\title{
Radio Channel Quality in Industrial Wireless Sensor Networks
}

\author{
Daniel Sexton \\ Phone 518-387-4121 \\ Email: sextonda@,crd.ge.com
}

\author{
Michael Mahony \\ Phone 518-387-5485 \\ Email:michael.mahony@crd.ge.com \\ GE Global Research \\ One Research Circle \\ Niskayuna, NY 12309 \\ Jay Werb \\ Sensicast System \\ 220-3 Reservoir Street \\ Needham, MA 02494 \\ Phone 781-453-2555 x203, Email: jwerb@sensicast.com
}

\author{
Michael Lapinski \\ Phone 518-387-6690 \\ Email:1apinski@crd.ge.com
}

\begin{abstract}
Wireless Mesh Sensor Networks are being deployed today in various monitoring and control applications. Some radio network designs, such as ZigBee, presume that radio connectivity is reasonably consistent over time. Others take the opposite approach of presuming that links are entirely unreliable, and build large degrees of physical redundancy into the network in the hope that a collection of redundant but unreliable individual links will result in a reliable overall system. Surprisingly little work has been done in the middle ground, endeavoring to understand the root cause of link failure in real-world factory environments and applying this knowledge in the design of protocols that adaptively detect and use workable radio channels.
\end{abstract}

In collaboration under a Department of Energy grant for the Industries of the Future, General Electric and Sensicast Systems have studied theoretical and actual performance of $2.4 \mathrm{GHz}$ IEEE 802.15.4 radio transceivers on the lab bench and on the factory floor, with particular attention to jamming from 802.11 and multipath fading. Temporal and frequency variations in link quality are explored. The implications for network reliability and protocol design are discussed.

\section{INTRODUCTION}

Wireless sensors for industrial applications are expected to open large opportunities for data collection where it has traditionally been considered technically impossible or cost prohibitive. To overcome installation and acceptance barriers a wide variety of requirements must be satisfied. Some of these barriers include cost and reliability. Short-range wireless technologies such as IEEE 802.15.4 [1] combined with mesh networking techniques are being widely considered as the answer to both cost and reliability in industrial settings. However RF communications, particularly indoors, is well known to be unpredictable.
Proper understanding of the channel characteristics is needed in order to determine adequate design margins to minimize the installation effort or the amount of physical network reconfiguration required as the environment around the network changes. One approach would simply be to over-configure the network by increasing the node density with additional mesh routing nodes. However this can cause issues with additional installation cost, network maintenance and decreased network capacity. A better approach would be to just slightly over-configure the network by understanding what the appropriate required design margins. Of course the success in this approach requires collection and analysis of a statistically relevant and representative set of RF channels and environments.

In our work on the Department of Energy grant we have attempted to better understand what effects are present in the $\mathrm{RF}$ environment in industrial facilities. As part of earlier work we have decided to focus our attention on the performance of 2.4 GHz IEEE 802.15.4 physical radios in this environment. With this information we hope to gain insight into the characteristics required of a mesh network as well as the suitable design margins required.

In our paper we present results of several types of measurements taken in several industrial facilities.

\section{OVERVIEW}

In our project several important technology and design decisions needed to be made early in our program so that we could launch critical design activities. One of these decisions was the choice of a physical radio technology. There were several options available to us. To make an initial decision we first took measurements of a readily accessible and representative harsh indoor environment. We took in initial channel measurement using a network analyzer and two 
antennas spaced 25 feet apart. An image of the facility is shown in Figure 1 and the schematic of the test equipment in shown in Figure 2. Initial measurements of this environment as shown in Figure 3 illustrates that in the $2.4 \mathrm{GHz}$ ISM band there is both significant frequency selective fading as well as flat fading depending on the area of interest. The dark points represent the actual measurement whereas the lighter points represent the fading as averaged over a $2 \mathrm{MHz}$ bandwidth. Clearly this initial measurement illustrates the need to utilize RF transmission techniques that can tolerate both frequency selective as well as flat fading.

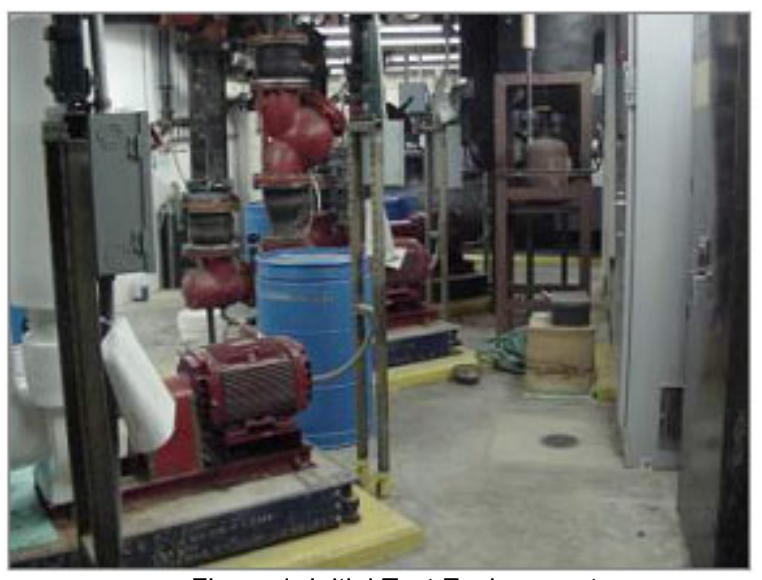

Figure 1: Initial Test Environment

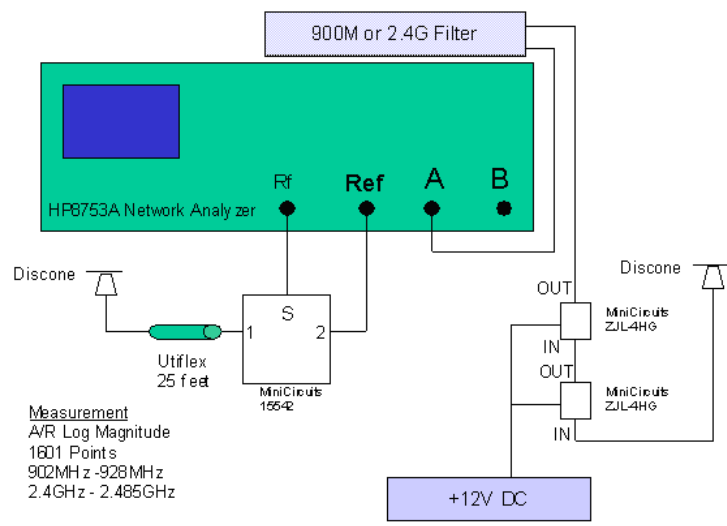

Figure 2: Initial Test Configuration

At the time of this experiment the first IEEE 802.15.4 Direct Sequence Spread Spectrum radios were being introduced and were an obvious consideration for this project.

We evaluated the ChipCon CC2420 [3] and decided to adopt this device for this project. With the transceiver output power of of $0 \mathrm{dBm}$ and the receiver sensitivity of $-94 \mathrm{dBm}$ the RF link budget could then be evaluated. However additional channel measurement data would be required to get a better understanding of the link margin requirements given a desired maximum range requirement of $100 \mathrm{~m}$ both line of sight and non line of sight. Figure 4 shows a plot of the first estimate of path loss using equation 1 , based on our initial data and a simple break point model we assume free space loss for the first $d=5 m$ and $n=3.5$ thereafter [13]. Note that for $0 \mathrm{dBm}$ output power we fall below the receiver sensitivity at about a $65 \mathrm{~m}$ distance using this path loss model.

$$
L_{o}=\left\{\begin{array}{cc}
40+20 \log (d) & d \leq 5 m \\
54+10 n \log \left(\frac{d}{5}\right) & d>5 m
\end{array}\right.
$$

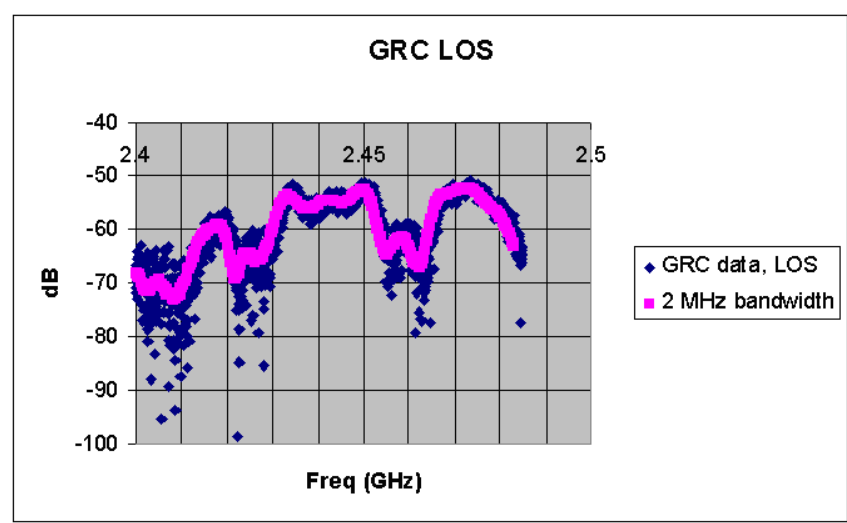

Figure 3: Channel Fading - Line of sight channel

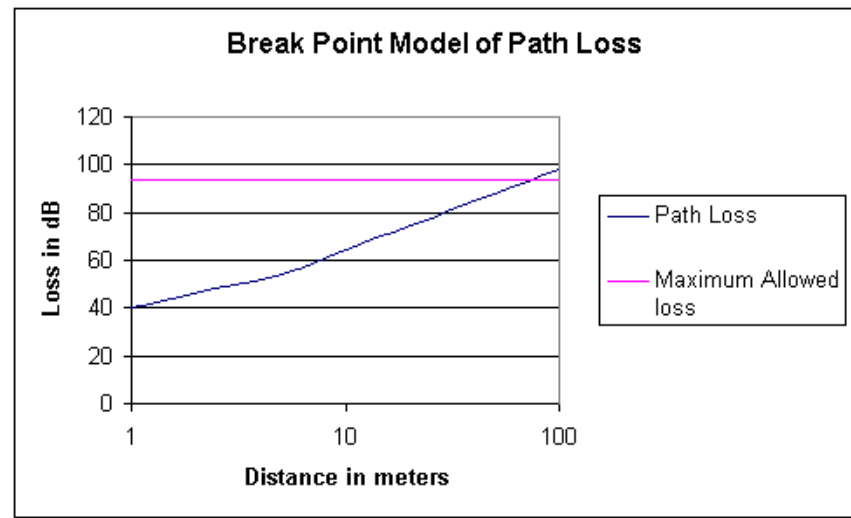

Figure 4: Breakpoint model for path loss

\section{CHANNEL MEASUREMENT STRATEGY}

With the radio selection made we took a focused strategy on channel measurements. We decided on a two-pronged approach to the collection of channel data. One approach used classical techniques for channel sounding. The other used the actual radios themselves to gather performance data. Relevant channel measurements made at ranges beyond $25 \mathrm{ft}$ were not practical with out initial measurement technique. We therefore choose to use the Spread Spectrum Sliding Correlator approach [2] for channel sounding. The test equipment we 
chose for these measurements was the Berkeley Varitronics Systems Raptor, which is well suited for indoor channel measurements [4]. The Raptor consists of a stand-alone direct sequence spread spectrum transmitter that has field-adjustable RF output power and selectable transmit frequency, and a mobile receiver that captures data using a PDA. In addition to the Raptor data, we characterized local noise sources in the 2.4GHz ISM band using a spectrum analyzer set for max hold to measure aggregate interference in the $83.5 \mathrm{MHz}$-wide band, and time-based, zero-span measurements of each IEEE 802.15.4 channel (see Figure 5)

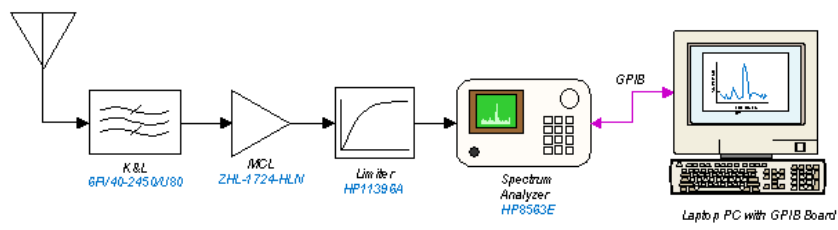

Figure 5: Spectrum Analyzer

The second measurement approach we used for channel characterization primarily consisted of a spectrum analyzer set for zero span and a CW RF signal source. . This measurement enabled us to extract the coherence time for the channel as well as calculate the fading statistics.

The third measurement approach used was to experiment with the performance of IEEE 802.15.4 radios in the actual industrial environment. To perform this test we constructed six units each of which housed a radio module and a single board computer with local data storage - Figure 6 . These units were placed at locations in industrial facilities where wireless sensors for equipment monitoring would typically be placed. The boxes then recorded the transmission performance for every packet sent on every channel so that a history of path performance could be determined. The data was then extracted from the units and stored in a database where it could be processed and sorted as desired.

The indoor channels that we measured displayed various amounts of interference from other radio systems. Although interference is not a topic for this paper it had a substantial effect on our measurements and had to be dealt with as the data was processed and analyzed.

The outcome of the coherence time measurements is an important discussion topic because it directly affects the channel stability and fading over time. In the channels we measured, channel coherence time averaged approximately $0.1 \mathrm{sec}-$ which represented people moving around within the channel environment, Figure 7. For many of the installations, the channel is relatively static; we are not considering mobile RF devices for our analysis at this time.

The bit rate for IEEE 802.15 .4 operating at $2.4 \mathrm{GHz}$ is $250 \mathrm{kbps}$ and in our system typical messages are in the range of 46 bytes including overhead, for a message duration of about $1.5 \mathrm{msec}$. With channel coherence time measured at about 100 msec, the channel can assumed to be stable over a single message and in fact can assumed to be stable over multiple transmissions of a message in the case of immediate retries.

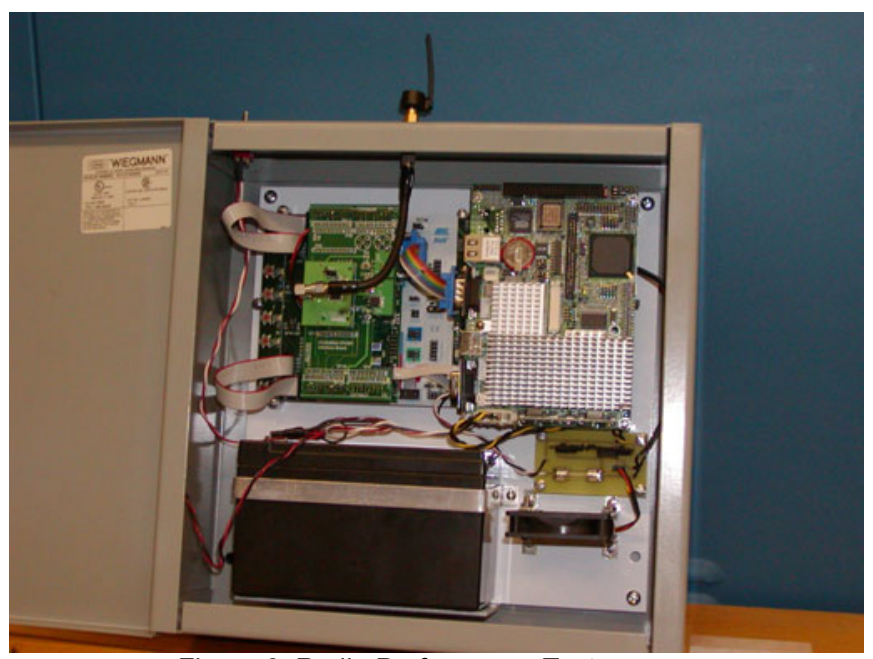

Figure 6: Radio Performance Tester

\section{MEASUREMENTS}

Off the shelf IEEE 802.15.4 radios currently do not have equalization built in. In industrial facilities with large metal surfaces the multipath delay spread may represent a problem. Andersen [5] reports that RMS delay spreads of 300ns might be expected and Ganesh [6] reports mean RMS delay spreads ranging as high as $73 \mathrm{nsec}$ ( $\max$ of $150 \mathrm{nsec}$ ). This amount of delay spread could cause a problem without equalization. In an IEEE 802.15.4 system with a chip rate of $2 \mathrm{Mc} / \mathrm{s}$ [1], an RMS delay spread of greater than $50 \mathrm{nsec}$ would be cause for concern. In 2002 we took extensive measurements of RMS delay spread and path loss for large industrial facilities that were housed in metal and concrete structures - in one example: a power generation facility with four GE Frame 7 turbines, two steam turbines and associated boilers. we calculated mean RMS delay spreads of $34.4 \mathrm{nsec}$ using the direct RF pulse method [2] which are consistent with the findings of Ganesh [6]. In the metallurgy facility at GE Global Research (a large metal and brick building) we measured RMS delay spreads of 10 to 200 nsec (with excess delay in the order of microsecnds) using the Raptor which is consistent with Andersen[5]. A recent presentation by Gorday [11] simulates IEEE 802.15.4 with various RMS delay spreads up to $500 \mathrm{nsec}$. This analysis shows that for an RMS delay spread of 400 nsec a degradation of about $17 \mathrm{~dB}$ might be experienced. We do not need to add additional margin for RMS delay spread on top of margin for fading as they are related. We do need to verify that we use the worst-case effect between the two. We needed to verify the fading characteristics of the channel to validate assumptions on fading and RMS delay spread. 


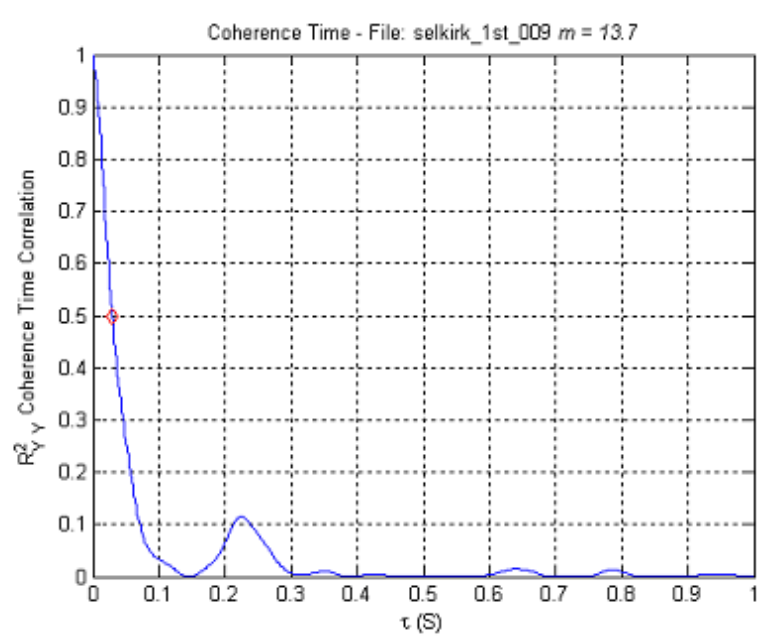

Figure 7: Channel Coherence Time Measurement

From measured data at various industrial locations, calculations of channel fading statistics varied substantially from location to location. Most of the measurements exhibited Nakagami-m characteristics while some exhibited Lognormal and Rayleigh characteristics. At this stage in the data collection it is unclear whether or not a single pdf can be used for the purposes of characterizing the channel. In general we have seen a large variance in the $\mathrm{m}$ - factor that represents the severity of fading, ranging from 0.9 to 55.9 (Figure 8 and Figure 9).

We are still in the process of collecting and characterizing channel data and cannot draw any definitive conclusions with the limited data set that we currently have. We will report our findings at a later date when the analysis is completed.

Even with channel fading data collected, having actual radio performance data will be useful to validate the characterization of the channels. At this point data collected from the actual radios using the units illustrated in Figure 5 has yielded the most interesting information. Again, we have not completely harvested all the information in this data but we have gathered some interesting results. In one experiment the boxes were distributed in a machine room (Figure 1) with a floor plan illustrated in Figure 10. Each of the locations for the units is marked 1 through 6 . The test was allowed to run for 4 hours. There is little to no motion in this installation as it is in an isolated area. During the experiment we collected lost packets, RSSI (received signal strength indicator) and LQI (link quality indicator) for each packet sent. Since the units are synchronized in time there can be no collisions. At any point in time only one unit was transmitting while the remainder were listening and recording. Transmission was then cycled to another unit until all had a time to transmit. This sequence occurred over and over again. The results of each transmission were recorded. Figure 12 plots the packet loss rate versus path and channel. The nomenclature for path is as follows: Path 12 represents the packet loss information for the path from unit 1 as the transmitter to unit 2 as the receiver and Path ${ }_{21}$ represents the reverse path. From this experiment we not only studied the performance of the radios but also the symmetry of the channel. In this experiment paths were both line of sight (LOS) as well as non line of sight (NLOS).

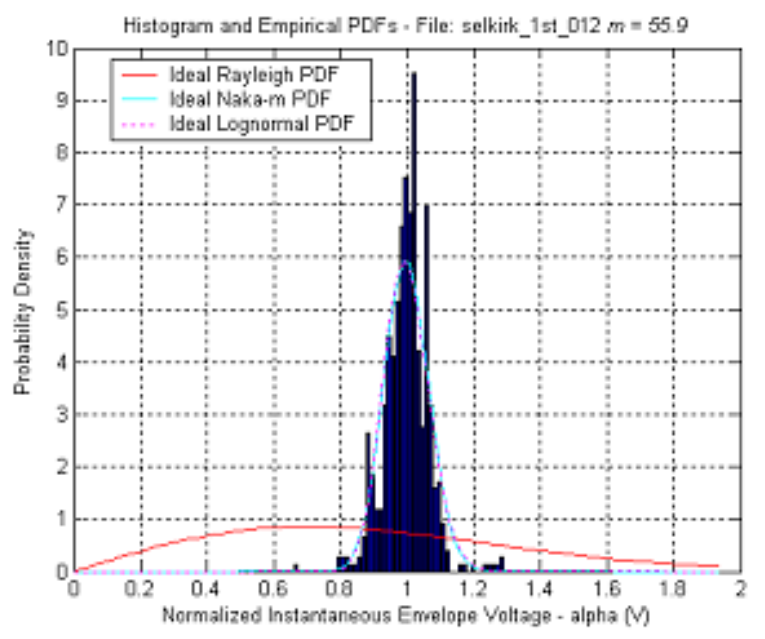

Figure: 8 Field data demonstrating a channel with Nakagami-m statistics showing a low degree of fading

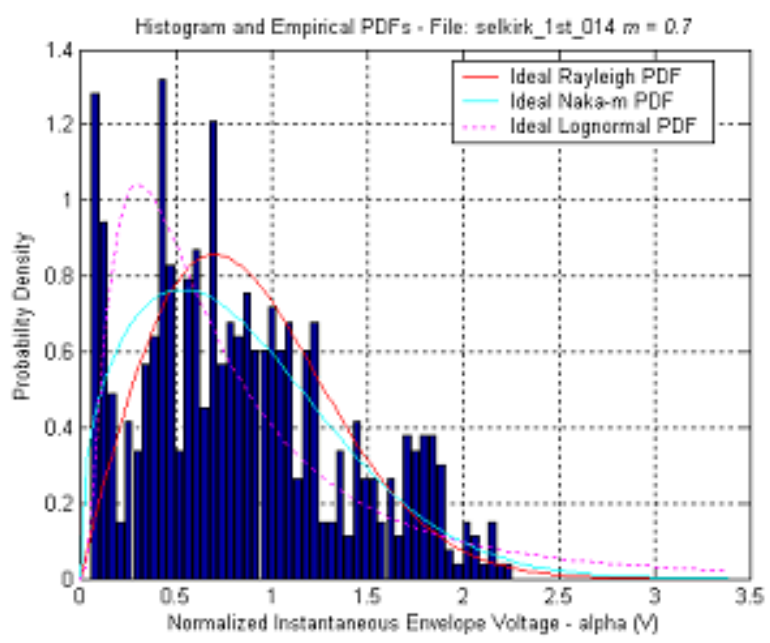

Figure 9: Field data demonstrating a channel with Nakagami-m statistics showing a high degree of fading

Figure 13 plots the same experiment in a different location - this data was collected in an industrial gas compression facility. Both data sets show rather vividly the effect of frequency selective fading. In this case of Figure 12 there was no channel that allowed for reliable communications over all paths for all units throughout the entire test period. In figure 13 only channel 15 was clear for all paths. None of the paths were very symmetric for all channels. The results of these experiments clearly show that a frequency agile approach or a multi-channel approach might be more robust than a single channel approach and is consistent with the initial measurements - Figure 3 . 


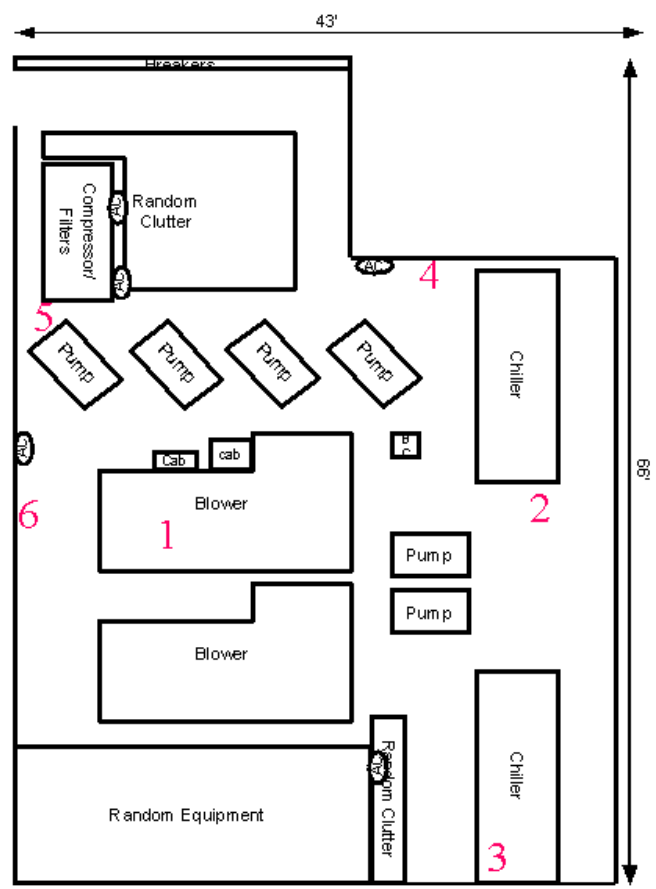

Figure 10: Machine room floor plan

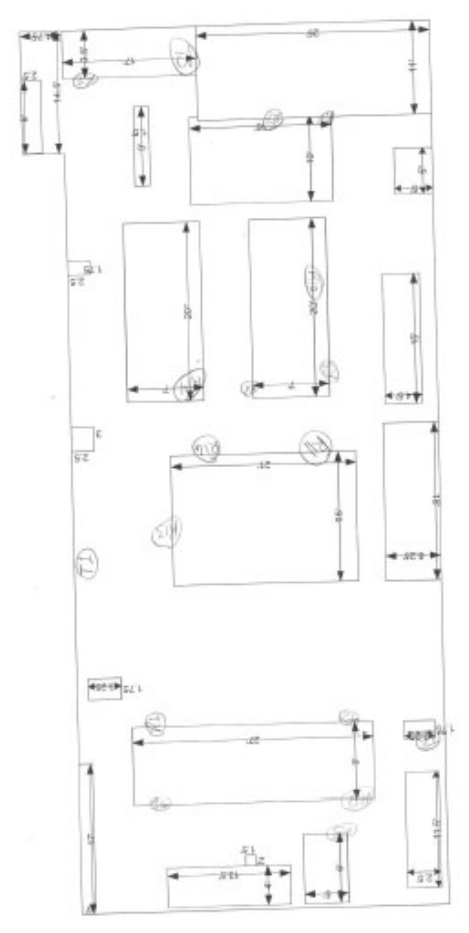

Figure 11: Compressor house floor plan

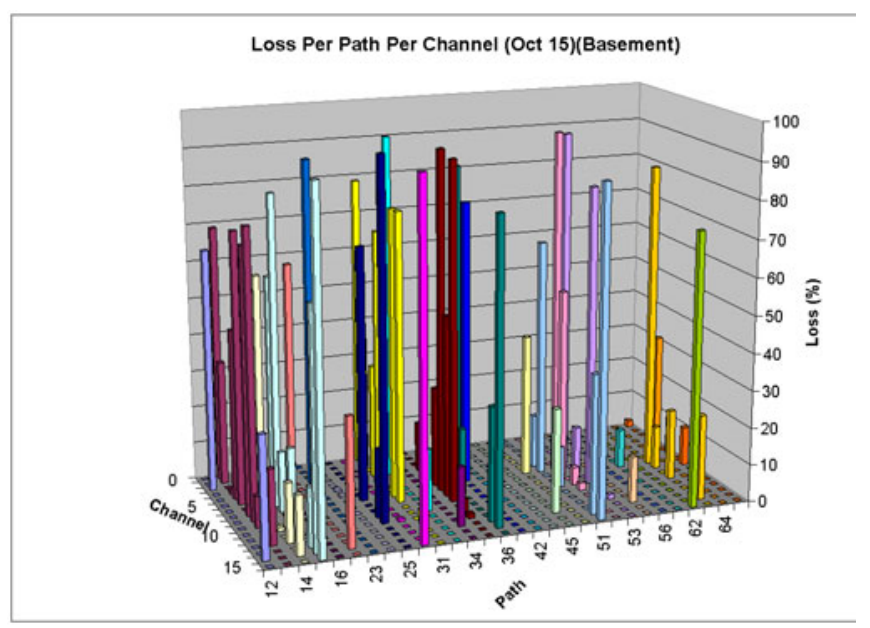

Figure 12: Packet loss versus path and channel - Machine Room

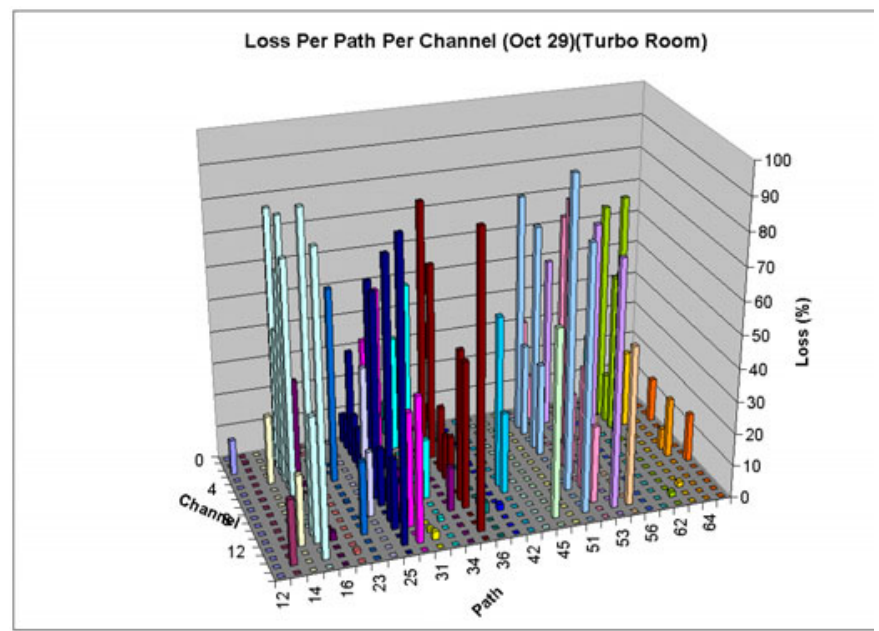

Figure 13: Packet loss versus path and channel - Compressor House

\section{CHANNEL FADE MARGIN}

Ultimately we need to determine the required fade margin or excess power required to obtain the desired reliability $(99 \%)$.

Free space channel path loss $(\mathrm{dB})$ is given by equation 2

$$
L_{o}=10 n \log \left(\frac{\lambda}{4 \Pi d_{o}}\right)
$$

Where $\lambda$ is the wavelength of the RF signal and $d_{O}$ is the path distance and $\mathrm{n}$ is the path loss exponent $(\mathrm{n}=2$ for ideal free space). From line of sight data measured in industrial facilities we estimated that $n=1.6$ gives a close approximation of the average attenuation. Our value of $\mathrm{n}$ was derived from averaged measurements from industrial facilities and is consistent with measurements reported by Andersen [5]. For non line of sight $\mathrm{n}=3.73$ is consistent as determined in reference [7].

Although these estimates for path loss give a good starting point for calculating the mean loss, the variance around that 
mean must also be considered. Link margin is required to overcome this variance and maintain a certain confidence level for the channel. Link margin can be gained by using multiple strategies. The three we choose to investigate are power amplification, spatial (path) diversity and frequency diversity. Both spatial and frequency diversity will provide a diversity gain that will allow us to reduce additional output power while maintaining our range.

In general we need to design our system for NLOS with multipath. Using a Rayleigh distribution as our model for the average receive power we will have the calculated mean power as defined above. To establish a goal for probability of success we also assume very slow fading or a static channel. We can assume that the distribution around the mean follows the Rayleigh pdf.

$$
f(r)=\frac{r}{\sigma^{2}} e^{\frac{r^{2}}{2 \sigma^{2}}} \quad r \geq 0 .
$$

If we assume that for any single transaction (which may involve multiple transmissions each of which is less than $4 \mathrm{msec}$ ) the channel is fixed then we can calculate the excess power required to guarantee with a $99 \%$ certainty that we will be at or above the expected power level from the following equation.

$$
P(R)=\int_{R}^{\infty} f(r) d r=e^{-\frac{R^{2}}{2 \sigma^{2}}}
$$

Where $\sigma^{2}$ is the time-averaged power received signal and $\mathrm{P}(\mathrm{R})$ is the probability of the received signal equal to or exceeding power level $R^{2}$. If $R^{2}$ is the power level we need for reliable transmission, then we can solve for $\sigma^{2}$ as the average power level, or we can solve for $\frac{\sigma^{2}}{R^{2}}$ as the static link margin $\mathrm{L}_{\mathrm{M}}$

$$
L_{m}=-10 \log (-2 \ln (P(R)))
$$

If we chose a $99 \%$ probability that we will obtain a particular power level, we will need to provide a $17 \mathrm{~dB}$ margin in our link budget (10dB for 95\%). This is consistent with the margin estimated for RMS delay spread [11] and with data measured in reference [7]. The link budget equation then becomes:

$$
\mathrm{M}_{\mathrm{e}}(\mathrm{dB})=\mathrm{T}_{\mathrm{p}}-\mathrm{L}_{\mathrm{o}}-\mathrm{L}_{\mathrm{m}}-\mathrm{R}_{\mathrm{s}}+\mathrm{G}_{\mathrm{ta}}+\mathrm{G}_{\mathrm{ra}}
$$

In our case we are using an omni-directional patch antennas that provide approximately $1 \mathrm{dBi}$. Our transmit power $\mathrm{T}_{\mathrm{p}}$ is $+15 \mathrm{dBm}$ and our receiver sensitivity is $-92 \mathrm{dBm}$ (typical). We use a slightly lower receiver sensitivity because of the losses associated with adding a power amplifier - these are measured values. If we calculate the link margin at $100 \mathrm{~m}$ NLOS (slow Rayleigh fading where the channel is considered stable and $\mathrm{n}=3.73$ ) our link margin requires $23 \mathrm{~dB}$ additional gain. Additional processing gain can be achieved through diversity as described in [12].

Refs [8] [9] [10] analyze the use of spatial diversity and frequency diversity techniques in terms of processing gain.
Todd [8] evaluated both spatial and frequency diversity at $1.7 \mathrm{GHz}$ and compared this to results obtain for $900 \mathrm{MHz}$ in the same environment. With spatial separations of 1 wavelength a diversity gain of $10.8 \mathrm{~dB}$ could be achieved with a frequency separation of $10 \mathrm{MHz}$ (two 802.15.4 channels). Combining these two diversity schemes, a total diversity gain of $16 \mathrm{~dB}$ was achieved [8] assuming a link availability of $99 \%$. One wavelength spatial diversity requires a spacing of $12 \mathrm{~cm}$ at $2.4 \mathrm{GHz}$, which in our application would make the overall package size too great. Large spatial diversity can be achieved by the use of multiple routing nodes either of which can receive and relay a message. Although this is not the same because of the significant path loss differences, it can be used to achieve additional diversity. For spatial diversity we can assume a worst-case analysis such that both receiving nodes are at the maximum distance of $100 \mathrm{~m}$. The result of ref [8] also effectively assumes that both receivers are at the same statistically average power point or at the same range and somewhat correlated.

In our system we will have a much higher diversity factor than two and our spatial separation is much greater. Until we collect more data we assume we can use uncorrelated channels. We therefore performed a simulation assuming totally independent uncorrelated channels for each level of diversity. If we consider our system to operate as a simple form of selection diversity [12] in a Rayleigh channel the probabilities multiply as the order of diversity $\mathrm{N}$ increases:

$$
P_{N}=\left(1-e^{-\frac{R^{2}}{2 \sigma^{2}}}\right)^{N}
$$

The results of this simulation are illustrated in Figure 14 and agree very closely to measured data as shown in Table 1 . Since we have 16 channels to choose from and we assume a minimum of two routing nodes available to each sensor node we have 32 degrees of freedom. Our simulation shows that in the best case we might expect a $23 \mathrm{~dB}$ gain from diversity with $99 \%$ availability in a pure Rayleigh channel; however it is doubtful we will ever achieve this amount of gain in practice. Coincidently to meet out link budget we need $23 \mathrm{~dB}$.

In this analysis we assume that when changing channels as well as changing paths we move to a totally independent yet statistically similar channel. In typical mesh routing networks, changing frequencies is much less costly than changing paths, as fewer routing nodes are needed. In addition, with frequency diversity all the routing tables remain stable and new routes do not need to be established. However in our network design we do keep a single alternate route in the routing tables if that route can be established.

No margin was added for interference due to coexistence issues, we are studying this and will be a subject for another paper. We do know that frequency diversity can be an effective tool in circumventing coexistence issues.

We do know from the data we have collected that we are getting significant advantage due to frequency diversity as shown in Figures 12 and 13, but we have not been able to extract the data to accurately quantify the diversity gains we 
are achieving. RSSI might be one valuable measure of this but we have no RSSI values for packets we did not receive. We do have RSSI as well as LQI values for both good and bad packets received and we will attempt to normalize those into information that will relate to diversity gain.

\begin{tabular}{|c|c|c|c|}
\hline Availability & Diversity & $\begin{array}{c}\text { Todd[8] } \\
\text { Maximum }\end{array}$ & Simulation \\
\hline $90 \%$ & 1 & $5.7 \mathrm{~dB}$ & $5.58 \mathrm{~dB}$ \\
\hline $99 \%$ & 1 & $10.8 \mathrm{~dB}$ & $10.62 \mathrm{~dB}$ \\
\hline $99 \%$ & 2 & $16.1 \mathrm{~dB}$ & $13.9 \mathrm{~dB}$ \\
\hline $99.9 \%$ & 1 & $16.9 \mathrm{~dB}$ & $14 \mathrm{~dB}$ \\
\hline \multicolumn{3}{|c}{ Table 1: Diversity Gain Comparison }
\end{tabular}

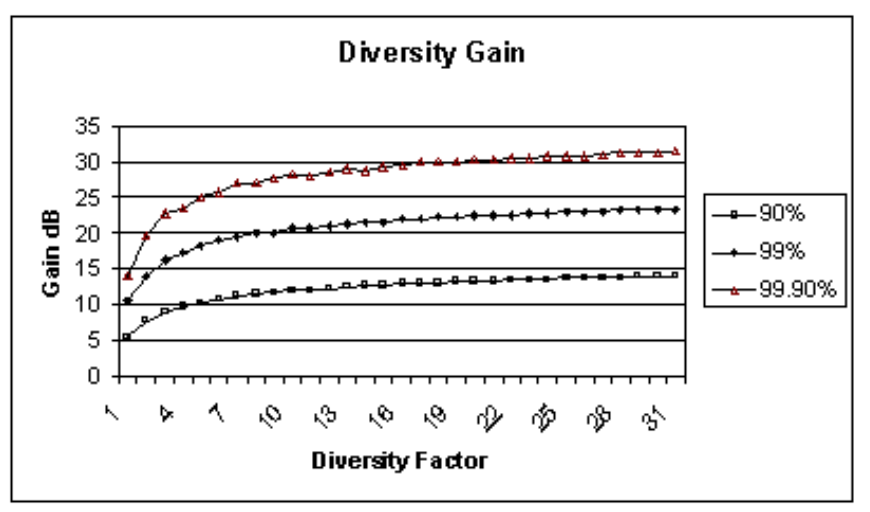

Figure 14: Estimated Diversity Gain

In our system we also require that a message is acknowledged and that the acknowledge is received to complete a transaction. (In a typical scenario, lost acknowledgements cause unnecessary retries, wasting power and bandwidth but not compromising reliability.) Currently the acknowledge must occur on the same channel and the reciprocal path as the original message. If there is minimal reciprocity in the paths then the diversity gain could be compromised. Our test data does not collect reciprocal path data within the coherence times we measured but we ran a correlation between the data to see if there was reciprocity anyway. For the machine room data in Figure 12 the correlation for reciprocity was 0.91 and for the compressor house data in Figure 13 the correlation for reciprocity was 0.87 both with a high confidence factor. With this level of correlation we can accept the diversity gains.

As previously mentioned, we have also seen Lognormal and Nakagami fading statistics. In line of sight channels we have measured exponential path loss factor of $n=1.6$. In these channels we expect to have an excess margin of $35 \mathrm{~dB}$ before fading effects are considered. This is $52 \mathrm{~dB}$ above our starting point for NLOS channels so we do not anticipate a problem. We will be analyzing the other statistic channel models in a similar way to determine the overall link margin choosing a conservative distribution.

We will be gathering additional data to validate the assumptions in this paper but the data we have already collected leads us to believe that we should have a high degree of confidence that we can maintain an adequate link margin to maintain our performance goals in all but the absolute worst case scenarios (all path distances $=100 \mathrm{~m}$ NLOS).

\section{CONCLUSIONS}

IEEE 802.15 .4 at $2.4 \mathrm{GHz}$ appears to be a suitable physical layer protocol for use in industrial environments; however much more testing and experience is needed. Power amplification beyond $15 \mathrm{dBm}$ may be required to achieve the desired range of $100 \mathrm{~m}$ NLOS, overcome the path loss uncertainty and keep network density low. Diversity schemes such as spatial diversity through mesh routing, and frequency diversity will significantly help to increase the reliability of the network by providing additional diversity gain. Channel reciprocity is also important in systems that require packet acknowledgements so that the full diversity gain can be achieved. In our network design we attempt frequency diversity before path diversity because of the lower cost.

\section{REFERENCES}

[1] IEEE Standard 802.15.4 - 2003, Standards for Telecommunications and Information Exchange Between Systems - Local Area and Metropolitan Area Networks - Specific Requirements Part 15.4: Wireless Media Access Control (MAC) and Physical Layer (Phy) Specifications for Low Rate Wireless Personal Area Networks (WPAN), Standard

[2] T. Rappaport. "Wireless Communications Principles and Practices," Book, 1996 by Prentice-Hill, Inc.

[3] ChipCon CC2420 Datasheet

[4] Berkeley Varitronics The Raptor Datasheet

[5] J. Andersen, T. Rappaport, "Propagation Measurements and Models for Wireless Communications Channels," IEEE Communications Magazine, January 1995.

[6] R. Ganesh, K. Pahlavan, "On The Modeling of Fading Multipath Indoor Radio Channels," IEEE GlobeCom 1989, Volume 3, November 1989, pages $1346-1349$.

[7] D. Cheung, C. Prettie, A Path Loss Comparison Between the $5 \mathrm{GHz}$ UNII Band (802.11a) and the 2.4GHz ISM Band (802.11b), Intel Labs Intel Corporation

[8] S. Todd, M. El-Tanny, S. Mahmoud, "Space and Frequency Diversity Measurements of the $1.7 \mathrm{GHz}$ Indoor Radio Channel Using a Four-Branch Receiver," IEEE Transactions on Vehicular Technology, Vol. 41, No 3. August 1992

[9] J. Wintersm J. Salz, R. Gitlin, "The Impact of Antenna Diverstiy on the Capacity of Wireless Communication Systems," IEEE Transactions on Communications, Vol 42. No. 2/3/4, February/March/April, 1994

[10] J. Winters, "The Diversity Gain of Transmit Diversity in Wireless Systems with Raleigh Fading," IEEE Transactions on Vehicular Technology, Vol 47, No 1, February 1998

[11] P. Gorday, 802.15.4 Multipath, doc: IEEE 802.15-04-0337-00-004b, July 2004

[12] D.G. Brennan, "Linear diversity combining techniques," Proc. IRE, vol. 47, pp. 1075-1102, June 1959

[13] IEEE 802.11.b Radio Interface, Radionet presentation. 